\section{Impactos da implementação dos dez passos da alimentação saudável para crianças: ensaio de campo randomizado}

\author{
Impacts of the 10 Steps to Healthy Feeding \\ in Infants: a randomized field trial
}

Márcia Regina Vitolo 1 Gisele Ane Bortolini 1 Carlos Alberto Feldens 2 Maria de Lourdes Drachler 3

\footnotetext{
1 Centro de Ciências da Saúde, Universidade do Vale do Rio dos Sinos, São Leopoldo, Brasil.

2 Departamento de Odontologia, Universidade Luterana do Brasil, Canoas, Brasil. 3 School of Allied Health Professions, University of East Anglia, Norwich, England.

Correspondência M. R. Vitolo

Centro de Ciências da Saúde, Universidade do Vale

do Rio dos Sinos.

C. P. 551, São Leopoldo, RS 93022-970, Brasil. vitolo@bios.unisinos.br
}

\begin{abstract}
This study assesses the impact of an intervention known as the Ten Steps to Healthy Feeding: A Nutritional Guide for Children under Two on nutritional conditions and infant health in low-income families. Two hundred newborns were randomized to the intervention group and three hundred to the control group. Parents of the intervention group received nutritional orientation during the child's first year of life. Both groups received visits at 6 and 12 months and routine follow-up by their pediatricians. The results ( $n=$ 397) showed that the intervention was associated with a higher proportion of exclusive breastfeeding at 4 months ( $R R=1.58$; 95\%CI: 1.21-2.06) and 6 months $(R R=2.34 ; 95 \% C I$ : $1.37-3.99)$ and breastfeeding at 12 months $(R R=1.26$; 95\%CI: 1.02-1.55) and a lower proportion of children with diarrhea ( $R R=0.68$; 95\%CI: 0.51-0.90), respiratory problems ( $R R=0.63$; 95\%CI: 0.46-0.85), use of medication ( $R R=0.56$; 95\%CI: 0.34-0.91), and dental caries ( $R R=0.56$; 95\%CI: 0.32-0.96) in the 12-16 month bracket. The intervention had no effect on the occurrence of anemia, hospitalization, or nutritional status. The results suggest that the nutritional orientation program led to positive changes in infant feeding practices and health conditions, but that it was insufficient to prevent iron deficiency anemia.
\end{abstract}

Child Welfare; Breast Feeding; Feeding

\section{Introdução}

O Guia Alimentar para Crianças Menores de 2 Anos 1 é uma iniciativa do Ministério da Saúde (Áreas Técnicas de Alimentação e Nutrição e da Saúde da Criança e Aleitamento Materno) e do Programa de Promoção e Proteção à Saúde da Organização Pan-Americana da Saúde (OPAS/ Brasil). Esse guia foi elaborado após amplo levantamento de dados existentes no país e complementados com a realização de estudos qualitativos, garantindo-se, assim, a identificação dos problemas existentes e, dentre eles, aqueles prioritários para intervenção. As bases científicas descritas no guia constituem sumário de ampla revisão da literatura internacional, baseadas no documento publicado pela OPAS/ Brasil 2, fundamentadas nos anais da reunião da Organização Mundial da Saúde/Fundo das Nações Unidas para a Infância sobre alimentação complementar realizada em Montpellier, na França, em dezembro de 1995, e publicados em 1998 3 3 . A partir desse estudo, foram elaboradas recomendações para uma alimentação saudável, expressas em Dez Passos para uma Alimentação Saudável: Guia Alimentar para Crianças Menores de Dois Anos, publicadas em um manual técnico para subsidiar os profissionais de saúde a promover práticas alimentares saudáveis para a criança pequena 4 .

As possibilidades de mudanças das práticas alimentares de crianças pequenas podem ser 
limitadas pelas condições sócio-econômicas e culturais, assim, sugere-se que, embora a alimentação humana possa referir-se à necessidade básica para a sobrevivência, ela não se limita a um fenômeno natural, na medida em que as práticas alimentares encontram-se vinculadas tanto à disponibilidade de alimentos quanto à escolha dos mesmos, dependendo dos valores culturais e das bases educacionais 1 .

O objetivo dessa investigação foi avaliar o impacto da aplicação das diretrizes nutricionais para crianças menores de dois anos estabelecidas pela Coordenação Geral da Política de Alimentação e Nutrição do Ministério da Saúde, por meio de um estudo de intervenção randomizado. As variáveis investigadas foram: duração do aleitamento materno exclusivo, freqüência de aleitamento total, ocorrência de morbidades, estado nutricional, anemia, cárie dentária e consumo de alimentos de baixo valor nutricional.

\section{Metodologia}

As crianças que participaram do estudo foram recrutadas no Hospital Centenário, o único da cidade de São Leopoldo, Rio Grande do Sul, Brasil, somente nos setores de atendimento do Sistema Único de Saúde (SUS). O cálculo do tamanho da amostra baseou-se em uma freqüência de aleitamento materno exclusivo até os quatro meses de $21,6 \%$ no grupo controle 5 e estimou uma diferença de $65,0 \%$ na freqüência dessa prática entre os grupos, após a intervenção. Outros parâmetros para esse cálculo foram: poder de $80 \%$ e nível de confiança de $95 \%$, o que determinou um tamanho amostral de 177 crianças em cada grupo, totalizando 354 crianças. Considerando uma previsão de perdas de $25,0 \%$, foram recrutados quinhentos pares mãe-filho para que o número amostral fosse atingido.

Estudantes de nutrição compareciam às enfermarias todos os dias da semana para identificar os recém-nascidos elegíveis para o estudo: peso de nascimento maior de 2.500 g, idade gestacional maior que 37 semanas. Os critérios de exclusão foram: mães HIV positivas, má-formação congênita, recém-nascidos encaminhados à Unidade de Terapia Intensiva (UTI) e parto múltiplo. As mães dos recém-nascidos que preenchiam os requisitos foram informadas sobre o estudo e convidadas a participar. Dos recém-nascidos elegíveis para o estudo, 90,0\% das mães aceitaram participar após a explica- ção das diferenças metodológicas entre os grupos controle e intervenção. As características sócio-demográficas das mães que recusaram participar do estudo não foram obtidas. Um pesquisador que não estava envolvido diretamente na seleção da amostra foi responsável pela randomização. A cada cinco mães que aceitavam participar da amostra, duas eram sorteadas para pertencer ao grupo intervenção, e as outras três alocadas para o grupo controle, e assim, consecutivamente, até ser atingido o número amostral estimado. A randomização em bloco foi usada para evitar ou diminuir possíveis desequilíbrios em algum ponto do processo de randomização. A proporção de pares mães/crianças maior no grupo controle foi estimada por considerar-se que o fato de a visita ocorrer somente seis meses após o contato na maternidade poderia acarretar maior perda desse grupo. Assim, após o processo de randomização, duzentos recém-nascidos foram incluídos no grupo intervenção, e trezentos, no grupo controle. Visitadores domiciliares (estudantes de graduação em nutrição) foram treinados para aplicar a intervenção dietética e realizar a coleta de dados.

Grupo Intervenção (I) - a intervenção consistiu de orientações dietéticas que compõem Dez Passos para uma Alimentação Saudável: Guia Alimentar para Crianças Menores de Dois Anos 4 dadas às mães durante 10 visitas domiciliares, realizadas nos primeiros 10 dias após o parto e depois mensalmente até aos 6 meses, aos 8, 10 e 12 meses. Os "dez passos" consistiram em: (1) dar somente leite materno até aos seis meses, sem oferecer água, chás ou qualquer outro alimento; (2) a partir dos seis meses, introduzir, de forma lenta e gradual, outros alimentos, mantendo o leite materno até aos dois anos de idade ou mais; (3) após seis meses, dar alimentos complementares (cereais, tubérculos, carnes, leguminosas, frutas e legumes) três vezes ao dia, se a criança receber leite materno; (4) a alimentação complementar deve ser oferecida sem que a rigidez de horários prejudique a sua ingestão; (5) a alimentação complementar deve ser espessa desde o início e oferecida de colher, começar com consistência pastosa (papas/purês) e, gradativamente, aumentar a consistência até chegar à alimentação da família; (6) oferecer à criança diferentes alimentos ao dia (uma alimentação variada é uma alimentação colorida); (7) estimular o consumo diário de frutas, verduras e legumes nas refeições; (8) evitar açúcar, café, 
enlatados, frituras, refrigerantes, balas, salgadinhos e outras guloseimas nos primeiros anos de vida e usar sal com moderação; (9) cuidar da higiene no preparo e manuseio dos alimentos, garantindo o armazenamento e conservação adequados; (10) estimular a criança doente e convalescente a alimentar-se, oferecendo seus alimentos preferidos, respeitando a sua aceitação.

Grupo Controle (C) - esse grupo recebeu visita aos 6 e aos 12 meses, quando foram coletados dados antropométricos, alimentares, sócio-demográficos e condições de saúde do lactente. Aos 6 meses, as entrevistadoras informavam as mães sobre os resultados antropométricos e orientavam-nas a procurar o serviço de saúde mais próximo, caso fosse detectado algum problema nutricional.

Aos 6 e aos 12 meses, entrevistadoras não envolvidas no processo de intervenção realizaram as visitas domiciliares para obtenção das variáveis do estudo. As entrevistadoras não eram cegas quanto ao grupo a que pertencia a criança.

O presente estudo investigou, para ambos os grupos, as seguintes variáveis aos 6 meses de idade: aleitamento materno exclusivo, definido como o uso de aleitamento materno como único alimento oferecido à criança, sem oferta de chá e água; aleitamento materno, definido como a presença de leite materno na alimentação da criança, independente da oferta de qualquer outro alimento; medidas antropométricas e as variáveis sócio-demográficas. Entre 12 e 16 meses de idade: medidas antropométricas - o peso (realizado por meio de balança digital) e comprimento foram obtidos de acordo com as recomendações internacionais. O estado nutricional das crianças foi indicado pelos índices de estatura para a idade e peso para estatura medido em escore-z do padrão americano National Center Health Statistics, utilizando-se o programa Epi Info versão 6.04. Para classificar baixo peso para a estatura $(\mathrm{P} / \mathrm{E}) \mathrm{e}$ baixa estatura para a idade (E/I), foi utilizado o ponto de corte <-2DP, e para classificar excesso de peso para a estatura, foi utilizado o ponto de corte $>+2 \mathrm{DP} 6$. Freqüência de morbidades no momento da entrevista, os pesquisadores perguntavam se, no mês anterior ou no momento atual, a criança apresentava febre que tenha necessitado medicamento, diarréia por mais de três dias, internação hospitalar e problemas respiratórios (tosse, coriza, obstrução nasal, respiração rápida ou difícil). Hemograma - as mães foram encaminhadas ao laboratório municipal da cidade, onde foi realizado o hemograma completo por meio de sistema automatizado (automação: Counter MDII). Os pontos de corte utilizados para diagnóstico de anemia e deficiência de ferro foram hemoglobina $<11 \mathrm{~g} / \mathrm{dl}$ e volume corpuscular médio $<74 \mathrm{ft}$, respectivamente. Exame clínico odontológico - foi realizado entre 12 e 16 meses de idade por um único examinador, treinado e calibrado (kappa $=0,88$ ), que não sabia a que grupo pertencia a criança. $\mathrm{O}$ exame foi realizado no centro de saúde com a criança posicionada em uma maca para crianças e sob luz natural. Foi utilizado critério visual com o auxílio de espelho bucal, após limpeza dos dentes com escova e secagem com gaze. A incidência de cárie precoce da infância, baseada na ocorrência de qualquer sinal clínico de cárie, incluindo mancha branca 7 e o número médio de superfícies cariadas (codificado como índice c-s 8 ) foram comparados entre os grupos. Consumo de alimentos industrializados - considerou-se a presença de refrigerante, chocolate, bala, salgadinhos, queijo petit suisse, mel e biscoito na alimentação, no mês anterior à última entrevista. Para a análise comparativa entre os grupos, com o objetivo de avaliar o impacto da intervenção, foi utilizada como critério a presença concomitante de refrigerante, chocolate, bala e salgadinho na alimentação das crianças. A idade em que foi introduzido o açúcar de adição foi obtida na última entrevista domiciliar. A partir da idade em que a criança recebeu chá, água, leite, sucos ou refrescos, perguntava-se sobre a adição de açúcar nesses líquidos. Adicionalmente, perguntava-se a idade que a criança tinha experimentado refrigerante, bala, doces, sorvetes ou qualquer outro alimento que contivesse açúcar.

O projeto de pesquisa Implementação e Avaliação do Impacto do Programa de Promoção para a Alimentação Saudável para Crianças Menores de Dois Anos foi aprovado pelo Comitê de Ética de Pesquisa da Universidade Federal do Rio Grande do Sul sob o número 200245. Foram obtidos os Termos de Consentimento Livre e Esclarecido para participar do estudo, realizar os exames laboratorial e odontológico.

Análise estatística - a homogeneidade da amostra quanto à distribuição das variáveis sócio-econômicas e demográficas entre os grupos intervenção e controle após a randomização foi examinada pelo teste qui-quadrado. Foram calculados os riscos relativos (RR) e respectivos intervalos de confiança de 95\% (IC95\%) para quantificar o efeito da intervenção sobre os desfechos de interesse: aleitamento materno, consumo de guloseimas, condições de morbidade e estado nutricional. O valor amostral diferiu, em cada análise, dependendo da disponibilidade dos dados. Considerou-se em 5\% o nível de rejeição da hipótese de nulidade $(\mathrm{p}<0,05)$. 


\section{Resultados}

A Figura 1 demonstra que 559 mães foram convidadas a participar da pesquisa para que o número amostral fosse atingido, havendo recusa de 59 mães, o que correspondeu a $10,5 \%$ das crianças elegíveis para o estudo. Das 500 crianças alocadas para os grupos intervenção ( $\mathrm{I}=$ 200) e controle $(C=300)$, não foi localizado o endereço de 3 e 28 crianças, respectivamente. No seguimento do estudo, as perdas foram de 34 crianças do grupo intervenção e de 38 do grupo controle. As causas de perdas foram: recusa em continuar participando do estudo ( $\mathrm{I}=$ 12; $C=6)$, mudança de endereço $(I=19 ; C=24)$, morte da mãe ou bebê $(I=1 ; C=2)$, doenças genéticas $(I=0 ; C=4)$, coleta de dados incom- pletos $(\mathrm{I}=0 ; \mathrm{C}=2)$, criança doada $(\mathrm{I}=1 ; \mathrm{C}=0)$ e doença da mãe $(I=1 ; C=0)$. As causas de perdas e suas proporções foram semelhantes entre os grupos. Completaram o estudo e tiveram seus dados analisados 397 crianças, 163 do grupo intervenção e 234 do grupo controle. O número amostral para o exame odontológico foi menor ( $n=376)$, pois 19 mães não compareceram à consulta odontológica agendada após a última visita domiciliar, e 2 crianças não apresentavam dentes no dia do exame.

A distribuição das variáveis sócio-econômicas familiares e do gênero das crianças foi semelhante para os grupos de controle e intervenção, confirmando a adequação do processo de randomização no início do estudo. Para ambos os grupos, intervenção e controle, $61,1 \%$ e

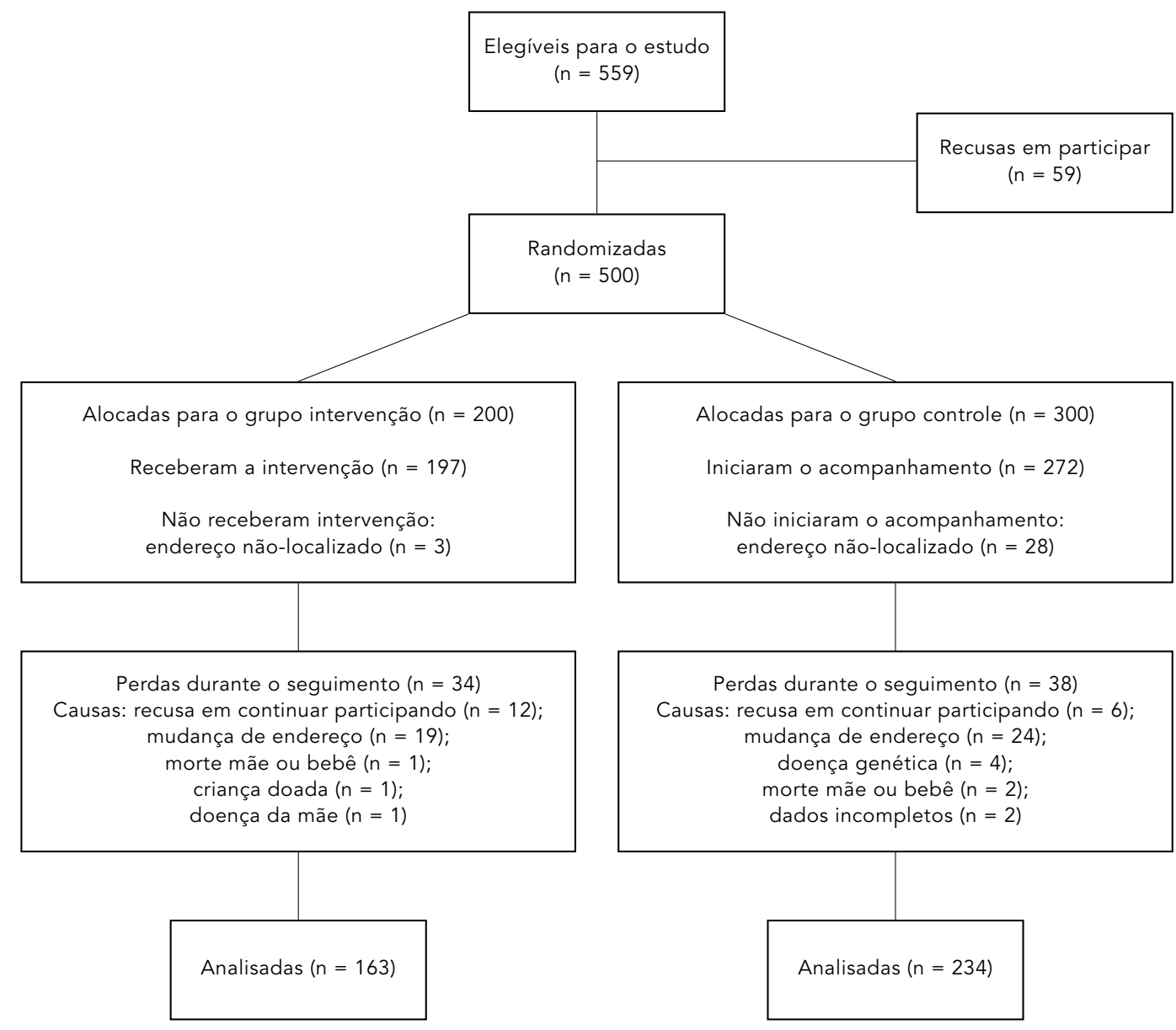


$56,0 \%$ das mães tinham até oito anos de escolaridade, respectivamente. Aproximadamente, $20,0 \%$ delas tinham menos de vinte anos de idade ao nascimento da criança, $15,0 \%$ viviam sem companheiro, e um terço tinha trabalho remunerado quando a criança tinha um ano de idade. A renda total da maioria das famílias era de até três salários mínimos, a escolaridade paterna, de até oito anos, e cerca de $10,0 \%$ dos pais estavam desempregados. A residência de um terço das crianças era de alvenaria, as demais eram de madeira ou mistas; cerca de 5,0\% dos domicílios tinham esgoto a céu aberto (Tabela 1). A concentração de habitantes por dormitório foi semelhante entre os grupos intervenção e controle (médias e desvios padrão 2,5 \pm 1,2 e $2,7 \pm 1,1$ respectivamente). A presença de pelo menos uma pessoa fumante no domicílio também foi semelhante entre os grupos $(42,6 \%$ no grupo intervenção e $38,2 \%$ no controle $\left(\chi^{2}, \mathrm{p}=\right.$ 0,380) (não apresentados em tabela).

Os resultados referentes aos desfechos alimentares mostraram que a proporção de crianças que receberam aleitamento materno exclusivo por menos de um mês foi estatisticamente maior no grupo controle, enquanto que as crianças do grupo intervenção apresentaram probabilidade $60,0 \%$ maior de amamentação exclusiva por quatro meses ou mais e mais do que o dobro da probabilidade de amamentação exclusiva aos seis meses de idade. A proporção de aleitamento materno aos 6 e 12 meses também foi maior para o grupo intervenção. O consumo de guloseimas (bala, refrigerante, salgadinho e chocolate) no primeiro ano de vida foi $40,0 \%$ menor no grupo intervenção. Os resultados referentes às condições de morbidade indicam que o grupo intervenção apresentou um risco $32,0 \%$ menor de diarréia, 37,0\% menor de doença respiratória e 44,0\% menor de uso de medicamentos. Não houve evidência de diferença entre os grupos para febre, internação hospitalar, anemia, baixa estatura e excesso de peso. A ocorrência de anemia foi alta, com mais de $60,0 \%$ das crianças de ambos os grupos apresentando valores de hemoglobina menor que $11 \mathrm{~g} / \mathrm{dl}$ e cerca da metade apresentando deficiência de ferro, demonstrada pelo valor de volume corpuscular médio menor do que $74 \mathrm{ft}$. A ocorrência de baixa estatura e de excesso de peso foi cerca de 5,0\% (Tabela 2). Não foi possível comparar os grupos em relação ao baixo peso, pois o número de crianças que apresentaram essa condição $(1,2 \%)$ foi muito pequeno (uma criança do grupo intervenção e seis do controle).

Das 376 crianças analisadas a partir do exame odontológico, 56 (14,9\%) apresentaram le- são de cárie. O número de superfícies atingidas variou de 1 a 10 , totalizando 195 . Os dentes mais afetados foram os incisivos superiores, correspondendo a $98,5 \%$ de todas as lesões observadas. A diferença observada entre a incidência de cárie no grupo intervenção $(10,2 \%)$ e grupo controle $(18,3 \%)$ indicou um risco de cárie $44,0 \%$ menor para as crianças do grupo intervenção (Tabela 2). O índice médio c-s foi significativamente menor ( $\mathrm{p}=0,006)$ no grupo intervenção $(0,37)$ quando comparado com o grupo controle $(0,63)$. Quanto às práticas alimentares cariogênicas (não apresentadas em tabelas), observou-se diferença estatística entre os grupos controle e intervenção. A introdução de açúcar foi mais tardia no grupo intervenção em relação ao grupo controle ( $\mathrm{p}=0,018)$. O consumo prévio de vários alimentos considerados prejudiciais à saúde bucal foi maior no grupo controle, sendo significante para biscoitos $(\mathrm{p}=0,000)$, queijo petit suisse ( $\mathrm{p}=0,001)$, mel $(\mathrm{p}=0,003)$, refrigerante $(\mathrm{p}=0,02)$ e bala $(\mathrm{p}=0,023)$.

\section{Discussão}

A comparação das variáveis sócio-demográficas familiares das crianças estudadas demonstrou semelhanças entre os grupos, permitindo a avaliação do efeito da intervenção.

As variáveis renda familiar e escolaridade materna e paterna exemplificam a condição sócio-econômica desprivilegiada da população estudada. No foco de políticas e programas em saúde pública, a melhor estratégia é priorizar boas condições de saúde para mulheres e crianças, visando romper o ciclo da pobreza e mánutrição ${ }^{9}$. Os resultados desse estudo mostraram que a intervenção aumentou a duração da amamentação exclusiva, a freqüência de aleitamento materno aos 12 meses de idade e diminuiu a ocorrência de morbidades. No Brasil, a média da prevalência de aleitamento materno exclusivo, entre as capitais brasileiras (dados de 1999), foi de cerca de $50,0 \%$ aos trinta dias de vida, sendo que, no quarto mês, a proporção foi de $21,6 \%$ do total, declinando para $9,7 \%$ no sexto mês 5 . É interessante relatar que taxas semelhantes foram observadas no grupo controle.

Estudo realizado na Índia, utilizando suplementação alimentar para crianças a partir de quatro meses de idade, demonstrou redução na duração do aleitamento materno e aumento na freqüência de morbidades, provavelmente pelo aumento do risco de contaminação alimentar, já que os suplementos exigiam adição de água 10 . De modo diferente, a intervenção do presente estudo foi apenas educativa, sem a 
Sexo da criança, variáveis maternas e sócio-econômicas para os grupos de intervenção e controle.

\begin{tabular}{|c|c|c|c|c|c|}
\hline \multirow{2}{*}{ Variáveis } & \multicolumn{2}{|c|}{ Intervenção } & \multicolumn{2}{|c|}{ Controle } & \multirow{2}{*}{ Valor de $p$} \\
\hline & $\mathrm{n}$ & $\%$ & $\mathrm{n}$ & $\%$ & \\
\hline \multicolumn{6}{|l|}{ Sexo da criança } \\
\hline Masculino & 93 & 57,1 & 130 & 55,5 & \\
\hline Feminino & 70 & 42,9 & 104 & 44,5 & 0,424 \\
\hline \multicolumn{6}{|c|}{ Escolaridade materna (anos) } \\
\hline$\leq 7$ & 99 & 61,1 & 131 & 56,0 & \\
\hline$\geq 8$ & 63 & 38,9 & 103 & 44,0 & 0,181 \\
\hline \multicolumn{6}{|l|}{ Idade materna (anos) } \\
\hline$\leq 19$ & 29 & 17,8 & 46 & 19,7 & \\
\hline $20-35$ & 116 & 71,2 & 168 & 71,8 & \\
\hline$>35$ & 18 & 11,0 & 20 & 8,5 & 0,416 \\
\hline \multicolumn{6}{|l|}{ Estado civil materno } \\
\hline Com companheiro & 139 & 85,3 & 193 & 83,2 & \\
\hline Sem companheiro & 24 & 14,7 & 39 & 16,8 & 0,340 \\
\hline \multicolumn{6}{|l|}{ Ocupação da mãe } \\
\hline Remunerada & 58 & 35,8 & 76 & 33,2 & \\
\hline Não remunerada & 104 & 64,2 & 153 & 66,8 & 0,334 \\
\hline \multicolumn{6}{|c|}{ Renda familiar (salários mínimos) } \\
\hline$\leq 1$ & 17 & 10,7 & 25 & 11,1 & \\
\hline $1-2,9$ & 101 & 63,5 & 132 & 58,7 & \\
\hline$\geq 3$ & 41 & 25,8 & 68 & 30,2 & 0,520 \\
\hline \multicolumn{6}{|c|}{ Escolaridade paterna (anos) } \\
\hline$\leq 7$ & 93 & 61,6 & 115 & 53,1 & \\
\hline$\geq 8$ & 58 & 38,4 & 98 & 46,0 & 0,091 \\
\hline \multicolumn{6}{|l|}{ Ocupação do pai } \\
\hline Remunerada & 130 & 88,4 & 195 & 91,1 & \\
\hline Não remunerada & 17 & 11,6 & 19 & 8,9 & 0,254 \\
\hline \multicolumn{6}{|l|}{ Tipo de casa } \\
\hline Madeira & 51 & 32,3 & 84 & 36,2 & \\
\hline Alvenaria & 84 & 53,2 & 106 & 45,7 & \\
\hline Mista & 23 & 14,5 & 42 & 18,1 & 0,332 \\
\hline \multicolumn{6}{|l|}{ Dejetos } \\
\hline Esgoto/fossa & 147 & 94,8 & 214 & 96,0 & \\
\hline Céu aberto & 8 & 5,2 & 9 & 4,0 & 0,390 \\
\hline
\end{tabular}

oferta de qualquer produto alimentar e com ênfase no aleitamento materno e na higienização durante o preparo da alimentação complementar, nono passo do guia alimentar 1. Em países em desenvolvimento, os substitutos alimentares do leite materno estão associados ao maior risco de morbi-mortalidade no primeiro ano de vida $11,12,13,14,15$.
A intervenção parece ter influenciado positivamente a amamentação e diminuído a ocorrência de morbidades, contudo, não houve evidência de efeito sobre a ocorrência de sobrepeso e baixa estatura, que se apresentou no grupo intervenção e controle em proporção semelhante ao observado em vários estudos nacionais ou de base populacional, os quais mos- 
Freqüências simples e percentuais, risco relativo (RR) e intervalo de confiança de 95\% (IC95\%) dos desfechos de acordo com o grupo intervenção e controle.

\begin{tabular}{|c|c|c|c|c|c|c|c|}
\hline \multirow[t]{2}{*}{ Variáveis } & \multicolumn{2}{|c|}{ Intervenção } & \multicolumn{2}{|c|}{ Controle } & \multirow[t]{2}{*}{$\mathbf{R R}$} & \multirow[t]{2}{*}{ IC95\% } & \multirow[t]{2}{*}{ Valor de $p$} \\
\hline & $\mathrm{n}$ & $\%$ & $n$ & $\%$ & & & \\
\hline \multicolumn{8}{|l|}{ Práticas alimentares } \\
\hline \multicolumn{8}{|l|}{$\begin{array}{l}\text { Aleitamento materno } \\
\text { exclusivo (meses) }\end{array}$} \\
\hline$<1$ & 54 & 33,3 & 111 & 48,0 & 0,69 & $0,54-0,90$ & 0,004 \\
\hline$\geq 4$ & 73 & 45,1 & 66 & 28,6 & 1,58 & $1,21-2,06$ & 0,001 \\
\hline$\geq 6$ & 31 & 19,1 & 19 & 8,2 & 2,34 & $1,37-3,99$ & 0,001 \\
\hline \multicolumn{8}{|l|}{ Aleitamento materno (meses) } \\
\hline Aos 6 & 114 & 66,3 & 134 & 55,6 & 1,19 & $1,02-1,39$ & 0,037 \\
\hline Aos 12 & 86 & 52,8 & 98 & 41,9 & 1,26 & $1,02-1,55$ & 0,040 \\
\hline Consumo de guloseimas* & 43 & 27,2 & 104 & 44,8 & 0,61 & $0,45-0,81$ & 0,001 \\
\hline \multicolumn{8}{|l|}{ Condições de morbidade } \\
\hline Diarréia & 46 & 28,4 & 98 & 42,0 & 0,68 & $0,51-0,90$ & 0,006 \\
\hline Febre & 77 & 47,5 & 115 & 49,3 & 0,96 & $0,78-1,19$ & 0,721 \\
\hline Problemas respiratórios & 42 & 25,8 & 96 & 41,0 & 0,63 & $0,46-0,85$ & 0,002 \\
\hline Uso de medicamento & 19 & 11,7 & 49 & 20,9 & 0,56 & $0,34-0,91$ & 0,017 \\
\hline Cárie dentária & 16 & 10,2 & 40 & 18,3 & 0,56 & $0,32-0,96$ & 0,030 \\
\hline Internação hospitalar & 9 & 5,5 & 15 & 6,4 & 0,86 & $0,38-1,91$ & 0,715 \\
\hline Hemoglobina $<11 \mathrm{~g} / \mathrm{dl}$ & 104 & 66,2 & 131 & 61,8 & 1,07 & $0,92-1,25$ & 0,380 \\
\hline $\mathrm{VCM}<74 \mathrm{ft}$ & 82 & 52,9 & 105 & 50,0 & 1,06 & $0,87-1,29$ & 0,584 \\
\hline \multicolumn{8}{|l|}{ Estado nutricional } \\
\hline Baixa estatura & 9 & 5,5 & 13 & 5,6 & 0,98 & $0,43-2,24$ & 0,980 \\
\hline Excesso de peso & 9 & 5,5 & 13 & 5,6 & 0,98 & $0,43-2,24$ & 0,988 \\
\hline
\end{tabular}

* guloseimas $=$ chocolate, bala, refrigerante e salgadinho. $\mathrm{VCM}=$ volume corpuscular médio.

tram índices de baixo peso, sobrepeso e baixa estatura semelhantes aos encontrados neste estudo 16,17,18,19,20,21,22. Estudo de Dewey et al. 22 , em Honduras, com o mesmo desenho metodológico, mostrou que a orientação para melhorar a qualidade da alimentação complementar entre 4 e 6 meses não modificou a situação do peso e da estatura aos 12 meses. A falta de impacto na proporção de crianças com baixa estatura pode refletir os efeitos crônicos de prejuízos nutricionais anteriores ao nascimento, mas outros estudos são necessários para confirmar essa hipótese.

A característica epidemiológica marcante do problema da desnutrição em crianças brasileiras passou a ser representada pelo retardo estatural, traduzindo os efeitos lentos, graduais e cumulativos do estresse nutricional, sobretudo nos dois primeiros anos de vida 23. Analisando o processo de transição nutricional que ocorre no país, parece surpreender, a princípio, a elevada prevalência de anemias. A análise de diversos estudos sugere as seguintes considerações: o declínio da desnutrição em crianças não foi acompanhado por melhoras no quadro da anemia ferropriva 24; não existem evidências de diferenças marcantes na ocorrência de anemia entre as diversas macrorregiões; embora seja assinalado um gradiente de prevalências em crianças de diferentes estratos sócioeconômicos, a anemia se difunde como um problema que afeta ricos e pobres 25. Entre 4 e 12 meses, a quantidade de reserva corporal de ferro aumenta para aproximadamente $130 \mathrm{mg}$, exigindo, portanto, fonte externa de ferro de alta biodisponibilidade 26 , já que o leite materno, quando associado a qualquer outro alimento, perde a alta biodisponibilidade do ferro, e, se esses alimentos não forem adequados, em pouco tempo, a criança vai desenvolver a deficiência de ferro. Em países desenvolvidos, a maioria dos estudos que comparou crianças em aleitamento materno exclusivo com crianças utilizando fórmula infantil ou alimentos comple- 
mentares fortificados com ferro não mostrou diferenças significativas no estado nutricional de ferro aos 6 meses de idade. Entretanto, na faixa etária entre 9 e 12 meses, o risco de deficiência de ferro em crianças amamentadas aumentou, quando não havia introdução de alimentos ricos em ferro 27,28. A baixa renda está associada à baixa reserva de ferro ao nascimento 29 , além de dificultar o acesso a alimentos complementares de qualidade, o que pode ter diminuído o impacto da intervenção dietética sobre a ocorrência de anemia aos 12 meses, resultado também corroborado por Childs et al. 30 .

A cárie dental entre 12 e 16 meses de idade foi uma das morbidades sobre a qual a intervenção dietética demonstrou ter efeito protetor, com incidência de cárie $44,0 \%$ menor no grupo intervenção comparado ao controle. Esse foi um efeito importante da intervenção, uma vez que a cárie é um problema de saúde pública que afeta bebês e crianças pré-escolares em todo o mundo, podendo levar a dor, dificuldades mastigatórias, problemas na função da fala, desordens gastrointestinais e problemas psicológicos 8,31,32. Além disso, lesão de cárie na dentição decídua é fator de risco para cárie na dentição permanente 33 . O efeito da intervenção em diminuir a ocorrência de práticas alimentares cariogênicas sugere que orientações para retardar a introdução do açúcar e evitar alimentos doces são potencialmente eficazes sobre o padrão alimentar no primeiro ano de vida. Essas práticas, provavelmente, contribuíram para a menor incidência de cárie nesse grupo, de forma direta ou indireta.

Os efeitos da intervenção sobre o comportamento alimentar indicam que houve adesão a práticas cuja implementação parece mais simples e objetiva, como retardar o uso de outros alimentos durante a amamentação e consumo de guloseimas e alimentos industrializados de baixo valor nutricional. É possível que as orientações dadas sistematicamente pelas pesquisadoras nos domicílios possa ter estimulado maior cuidado e preocupação com os aspectos alimentares e higiênicos do lactente, contribuindo para os benefícios encontrados.

Os aconselhamentos dietéticos esbarram em uma série de limitações como a complexidade da mudança comportamental, os aspectos culturais e o baixo poder aquisitivo. Santos et al. 34 desenvolveram projeto para verificar o impacto de orientações dietéticas na condição nutricional de lactentes. Os achados não foram significativos, exceto o maior valor do índice de peso para a estatura no grupo intervenção. Naquele estudo, porém, a captação das crianças variou de 8 dias a 180 dias, não sendo possível avaliar o impacto sobre a duração do aleitamento materno, diferentemente deste estudo em que todas as crianças foram randomizadas a partir do nascimento.

Entre as limitações do presente estudo, deve destacar-se que a ausência de cegamento dos entrevistadores na coleta de dados pode constituir-se um viés, uma vez que o conhecimento quanto ao grupo a que pertencia a criança poderia modificar a abordagem do entrevistador quanto às práticas alimentares e ocorrência de morbidades. É importante ressaltar, entretanto, que o cegamento, mesmo que previsto na metodologia, não seria completo, pois as mães que estariam recebendo visitas domiciliares desde o nascimento do bebê seriam facilmente identificadas nos primeiros momentos da entrevista. Além disso, deve considerar-se a possibilidade de um efeito psicológico benéfico de pertencer ao grupo intervenção (Hawthorne), sendo impossível separar o efeito da orientação nutricional da atenção oferecida às mães durante as visitas. Deve ressaltar-se, no entanto, que, em estudos de intervenção sobre comportamento alimentar, não é possível cegar os indivíduos e entrevistadores 35 .

As políticas públicas em saúde são diretrizes de ações que devem estar de acordo com as realidades da comunidade e serem capazes de prevenir ou solucionar os problemas atuais no curto prazo, mesmo antes da efetivação de reformas econômicas e sociais de maior impacto. A implementação dos Dez Passos para uma Alimentação Saudável: Guia Alimentar para Crianças Menores de Dois Anos mostrou-se efetiva na melhora de alguns aspectos da saúde da criança (aleitamento materno, práticas alimentares e morbidades), sendo evidente a necessidade da ampla aplicação desse programa na rede básica de saúde e na comunidade. Outras providências devem ser urgentemente tomadas quanto à situação da anemia infantil que atinge atualmente cifras alarmantes, como, por exemplo, a suplementação profilática de ferro e o enriquecimento dos alimentos. Tais medidas já se mostraram efetivas quando implementadas de forma sistemática e contínua 36,37,38,39. As crianças na faixa etária menor de dois anos beneficiam-se mais intensamente com programas que priorizam a vigilância nutricional no pré-natal e no primeiro ano de vida. 


\section{Resumo}

Efeitos de uma intervenção baseada na implementação dos Dez Passos para uma Alimentação Saudável: Guia Alimentar para Crianças Menores de Dois Anos sobre as condições nutricionais e de saúde de lactentes em famílias de baixa renda. Randomizou-se duzentos recém-nascidos para o grupo intervenção e trezentos para o grupo controle. O grupo intervenção recebeu orientações dietéticas no primeiro ano de vida. Os dois grupos receberam visitas aos 6 e 12 meses e acompanhamento de rotina pelos seus pediatras. Os resultados ( $n=397)$ mostraram que a intervenção associouse a maior proporção de aleitamento materno exclusivo aos 4 meses ( $R R=1,58$; IC95\%: 1,21-2,06) e 6 meses $(R R=2,34$; IC95\%: 1,37-3,99) e amamentadas aos 12 meses ( $R R=1,26$; IC95\%: 1,02-1,55) e a menor proporção de crianças que apresentaram diarréia $(R R=0,68$; IC95\%: 0,51-0,90), problemas respiratórios $(R R=0,63$; IC95\%: 0,46-0,85), uso de medicamentos $(R R=0,56$; IC95\%: 0,34-0,91) e cárie dental $(R R=0,56$; IC95\%: 0,32-0,96) na faixa etária de 12 a 16 meses. Não houve efeito da intervenção sobre a ocorrência de anemia, internações hospitalares e estado nutricional. Os resultados sugerem que o programa de orientações dietéticas promoveu modificações positivas nas práticas alimentares e nas condições de saúde da criança, porém foi insuficiente para prevenir a anemia ferropriva.

Bem-estar da Criança; Aleitamento Materno; Alimentação

\section{Colaboradores}

M. R. Vitolo realizou a definição logística e o treinamento da coleta de dados, além da organização, preparação e discussão dos resultados. G. A. Bortolini supervisionou a coleta de dados em campo, organizou o banco de dados, realizou a busca bibliográfica e formatou o artigo. C. A. Feldens colaborou na coleta de dados em campo e no preparo do texto do artigo; foi responsável por todos os dados referentes à cárie dentária. M. L. Drachler participou do treinamento na coleta de dados e foi responsável por toda a análise estatística e montagem das tabelas.

\section{Agradecimentos}

Este estudo recebeu apoio financeiro do Conselho Nacional de Desenvolvimento Científico e Tecnológico (CNPq). Agradecemos as coordenações técnicas da Secretaria Municipal de Saúde de São Leopoldo, Rio Grande do Sul, Brasil, pela oportunidade e apoio recebidos no decorrer do projeto.

\section{Referências}

1. Ministério da Saúde. Guia alimentar para crianças menores de 2 anos. Brasília: Ministério da Saúde, 2002.

2. Giugliani ERJ, Victora CG. Normas alimentares para crianças brasileiras menores de dois anos. Embasamento científico. Brasília: Organização Pan-Americana da Saúde, Organização Mundial da Saúde; 1997.

3. World Health Organization. Complementary feeding of young children in developing countries: a review of current scientific knowledge. Geneva: World Health Organization; 1998.

4. Ministério da Saúde. Dez passos para uma alimentação saudável: guia alimentar para crianças menores de dois anos. Brasília: Ministério da Saúde/Organização Pan-Americana da Saúde; 2002.

5. Ministério da Saúde. Prevalência do aleitamento materno nas capitais brasileiras e Distrito Federal. Brasília: Ministério da Saúde; 2001.

6. World Health Organization. Physical status: the use and interpretation of anthropometry. Geneva: World Health Organization; 1995. (Technical Report Series 854).

7. Ismail AI. Prevention of early childhood caries. Community Dent Oral Epidemiol 1998; 26 Suppl 1:49-61.

8. Mattos-Graner RO, Zelante F, Line RC, Mayer MP. Association between caries prevalence and clinical, microbiological and dietary variables in 1.0 to 2.5-year-old Brazilian children. Caries Res 1998; 32:319-23.

9. Nantel G, Tontisirin K. Policy and sustainability issues. J Nutr 2002; 132:839-44.

10. Bhandari N, Bahl R, Nayyar B, Khokhar P, Rohde J, Bhan, MK. Food supplementation with encouragement to feed it to infants from 4 to 12 months of age has a small impact on weight gain. J Nutr 2001; 13:1879-80.

11. Victora CG, Vaughan JP, Lombarda C, Funchs SMC, Gigante LP, Smith PG, et al. Evidence for protection by breast-feeding against infants deaths from infectious diseases in Brasil. Lancet 1987; 2:317-22.

12. Howie PW, Forsyth JS, Ogston SA, Clark A, Florey CD. Protective effect of breastfeeding against infection. BMJ 1990; 300:11-6.

13. World Health Organization. Collaborative Study Team on the Role of Breastfeeding on the Prevention of Infant Mortality: how much does breastfeeding protect against infant and child mortality due to infections diseases: a pooled analysis of six studies from less developed countries. Lancet 2000; 355:451-5.

14. Passos MC, Lamounier JA, Alves J, Silva CAM, Freitas SN, Baudson MFR. Práticas de amamentação no Município de Ouro Preto, MG, Brasil. Rev Saúde Pública 2000; 34:617-22.

15. Giugliani ERJ. O aleitamento materno na prática clínica. J Pediatr (Rio de J) 2000; 76:238-52.

16. Monteiro CA, Conde WL. Tendência secular da desnutrição e da obesidade na infância na cidade de São Paulo (1974-1996). Rev Saúde Pública 2000; 34 Suppl 6:52-61. 
17. Post LA, Victora CG. The low prevalence of weightfor-height deficits in Brazilian children is related to body proportions. J Nutr 2001; 131:1133-4.

18. Motta MEFA, Silva GAP. Desnutrição e obesidade em crianças: delineamento do perfil de uma comunidade de baixa renda. J Pediatr (Rio de J) 2001; 77:288-92.

19. Wang Y, Monteiro C, Popkin BM. Trends of obesity and underweight in older children and adolescents in the United States, Brazil, China, and Russia. Am J Clin Nutr 2002; 75:971-7.

20. Strufaldi MWL, Puccini RF, Pedroso GC, Silva EMK, Silva NN. Prevalência de desnutrição em crianças residentes no Município de Embu, São Paulo, Brasil, 1996-1997. Cad Saúde Pública 2003; 19:421-8.

21. Drachler ML, Macluf SPZ, Leite JCC, Aerts DRGC, Giugliani ERJ, Horta BL. Fatores de risco para sobrepeso em crianças no Sul do Brasil. Cad Saúde Pública 2003; 19:1073-81.

22. Dewey KG, Cohen RJ, Brown KH, Rivera LL. Age of introduction of complementary foods and growth of term, low-birth-weight, breast-fed infants: a randomized intervention study in Honduras. Am J Clin Nutr 1999; 69:679-86.

23. Batista Filho ME, Rissin A. A transição nutricional no Brasil: tendências regionais e temporais. Cad Saúde Pública 2003; 19 Suppl 1:181-91.

24. Barreto ML, Carmo EH. Determinantes das condições de saúde e problemas prioritários no país. 11ạ Conferência Nacional de Saúde, 2000. http:// www.saude.gov/11cns/index-11cns.htm (acessado em 05/Mar/2004)

25. Osório MM, Lira PI, Batista Filho M, Ashworth A. Prevalence of anemia in children 6-59 months old in the state of Pernambuco, Brazil. Rev Panam Salud Pública 2001; 10:101-7.

26. Wharton B. Iron deficiency in children: detection and prevention. Br J Haematol 1999; 106:270-80.

27. Siimes MA, Salmenpera L, Perheetupa J. Exclusive breast-feeding for nine months: risk of iron deficiency. J Pediatr 1994; 104:196-9.

28. Duncan B, Schifman RB, Corrigan JJ, Schaefer C. Iron and the exclusively breast-fed infant from birth to six months. J Pediatr Gastroenterol Nutr $1995 ; 4: 421-5$.
29. Sipinilo A, Capuzzo E, Piazzi G, Incola S, Colonna L, Iasci A. Maternal hight-risk factors and severity of growth deficit in small for gestacional age infants. Early Hum Dev 1994; 38:35-43.

30. Childs F, Aukett A, Darbyshire P, Ilett S, Livera LN Dietary education and iron deficiency anemia in the inner city. Arch Dis Chil 1997; 76:144-52.

31. Ramos-Gomez FJ, Weintraub JA, Gansky SA, Hoover CI, Featherstone JD. Bacterial, behavioral and environmental factors associated with early childhood caries. J Clin Pediatr Dent 2002; 26:16573.

32. Berkowitz RJ. Causes, treatment and prevention of early childhood caries: a microbiologic perspective. J Can Dent Assoc 2003; 69:304-7.

33. Li Y, Wang W. Predicting caries in permanent teeth from caries in primary teeth: an eight-year cohort. J Dent Res 2002; 81:561-6.

34. Santos I, Victora CG, Martines J, Gonçalves H, Gigante DP, Valle NJ, et al. Nutrition counseling increases weight gain among Brazilian children. J Nutr 2001; 131:2866-73.

35. Fletcher RH, Fletcher SW, Wagner EH. Clinical epidemiology: the essentials. 3rd Ed. Baltimore: Williams \& Wilkins; 1996

36. Stekel A, Olivares M, Cayazzo M, Chadud P, Llaguno S, Pizarro F. Prevention of iron deficiency by milk fortification. II. A field trial with a full-fat acidified milk. Am J Clin Nutr. 1988; 47:265-9.

37. Hertrampf E, Olivares M, Walter T, Pizzaro F, Heresi G, Llaguno S, et al. Anemia ferropriva en el lactente: erradication com leche fortificada con hierro. Rev Médica Chilena 1990;118:1330-7.

38. Walter T, Dallman PR, Pizzaro F, Velozo L, Pena G, Bartholmey SJ, et al. Effectiveness of iron-fortified infant cereal in prevention of iron deficiency anemia. Pediatrics 1993; 91:976-82.

39. Muliis RM, Owen A, Blaskovich L. National action conference on healthy eating for children: a policy dialogue. J Nutr Educ 1995; 27:222-34.

Recebido em 29/Jun/2004

Versão final reapresentada em 24/Mar/2005

Aprovado em 08/Abr/2005 